\title{
Feasibility of Pulse Oximetry Pre-discharge Screening Implementation for detecting Critical Congenital heart Lesions in newborns in a secondary-level maternity hospital in the Western Cape, South Africa: The 'POPSICLe' study
}

\author{
A M van Niekerk, ${ }^{1}$ BSc, MB ChB, DCH (SA), FCPaed (SA), Cert Cardiol Paed (SA); R M Cullis, ${ }^{1}$ MB ChB, DCH (SA), FCPaed (SA); \\ L L Linley, ${ }^{1}$ MB ChB, FCPaed (SA), Reg Neon (SA); L Zühlke, ${ }^{2}$ MB BCh, DCH, FCPaed (SA), Cert Cardiol Paed (SA), MPH, FESC, PhD (Paed) \\ ${ }^{1}$ Division of Neonatal Medicine, School of Child and Adolescent Health, Faculty of Health Sciences, University of Cape Town; Mowbray \\ Maternity Hospital, Cape Town, South Africa \\ ${ }^{2}$ Department of Paediatrics, Red Cross War Memorial Children's Hospital and Faculty of Health Sciences, University of Cape Town, \\ South Africa
}

Corresponding author: A M van Niekerk (anika.vanniekerk@westerncape.gov.za)

\begin{abstract}
Background. Early detection of critical congenital heart disease (CCHD) through newborn pulse oximetry (POx) screening is an effective strategy for reducing paediatric morbidity and mortality rates and has been adopted by much of the developed world.

Objectives. To document the feasibility of implementing pre-discharge POx screening in well babies born at Mowbray Maternity Hospital, a busy government hospital in Cape Town, South Africa. Parent and staff acceptance was assessed.

Methods. We conducted a prospective study of predischarge POx screening in one postnatal ward, following informed parental consent. Results. During the 4-month study period, 1017 of 2256 babies discharged (45.1\%) were offered POx screening and 1001 were screened; $94.0 \%$ of tests took $<3$ minutes to perform, $4.3 \% 3-5$ minutes and $1.7 \%>5$ minutes. Eighteen patients needed second screens and three required third screens. Only 3.1\% protocol errors were made, all without consequence. The vast majority (91.6\%) of nursing staff reported insufficient time to perform the study screening in addition to their daily tasks, but $\sim 75 \%$ felt that with a full nursing staff complement and if done routinely (not part of a study), pre-discharge POx screening could be successfully instituted at our facility. Over $98 \%$ of the mothers had positive comments. Two babies failed screening and required echocardiograms; one was diagnosed with CCHD and the other with neonatal sepsis. The sensitivity and specificity were 50\% (95\% confidence interval (CI) 1.3 - 98.7\%) and 99.9\% (95\% CI 99.4 - 100\%), respectively, with a percentage correct of $99.8 \%$. Conclusions. POx screening was supported and accepted by staff and parents. If there are no nursing staff shortages and if it is done routinely before discharge, not as part of a study, we conclude that POx screening could be implemented successfully without excessive false positives or errors, or any additional burden to cardiology services.
\end{abstract}

S Afr Med J 2016;106(8):817-821. DOI:10.7196/SAMJ.2016.v106i8.10071

Congenital heart disease (CHD) is common and accounts for more deaths than any other type of congenital malformation. The incidence of significant CHD is said to be 8 - 9 per 1000 live births and that of critical congenital heart disease (CCHD), which leads to death or needs surgical intervention before 28 days, approximately 2 - 3 per $1000 .^{[1,2]}$ These incidences are constant worldwide, across geographical and ethnic backgrounds and in spite of variations in socioeconomic conditions, ${ }^{[3]}$ but are thought to be underestimated in countries where antenatal booking is delayed or prenatal ultrasound screening not uniformly practised. ${ }^{[4]}$ Early diagnosis currently relies on antenatal fetal anomaly screening and neonatal examination. In South Africa (SA), the vast majority of congenital cardiac lesions are not diagnosed before birth owing to limited antenatal screening for CHD.

Cardiac services have improved, and most newborns with CCHD can be diagnosed with echocardiography, stabilised with prostaglandin and treated with surgery or trans-catheter intervention. ${ }^{[5]}$ Without an early diagnosis and appropriate treatment, half of those born with significant CHD will die in infancy or early childhood, a third of them within the first month of life. ${ }^{[4,6]}$

Newborn pulse oximetry (POx) is the first appropriately simple method for universal screening for $\mathrm{CHD},{ }^{[7]}$ the earliest reports having been published in 1995. The rationale is that most CCHD results in a degree of hypoxaemia that would not necessarily produce visible cyanosis and would therefore not be clinically detectable. There is significant evidence that early detection of CCHD through POx monitoring is an effective strategy for reducing morbidity and mortality rates in young children. ${ }^{[8-11]}$ In 2009 , the American Heart Association (AHA) and the American Academy of Pediatrics (AAP) released a scientific statement concluding that 'methods to improve the early detection of CCHD appear warranted' and called for larger population-based studies on implementation. ${ }^{[5]}$

In 2011, the AAP and the Secretary for Health and Human Services recommended the addition of POx screening to routine newborn screening to detect CCHD in the USA. Most US states have adopted POx screening into routine newborn screening. ${ }^{[12]}$ Many European multicentre studies have been performed, several European countries screen routinely, and the UK is trialling implementation on a large scale. ${ }^{[8-10,13]}$ In a large cost-effectiveness analysis, Ewer et al. ${ }^{[14]}$ concluded that '... pulse oximetry is a simple, safe, feasible test that is acceptable to parents and staff and adds value to existing screening. It is likely to identify cases of CCHDs that would otherwise go undetected. It is also likely to be cost-effective given current acceptable thresholds. The detection of other pathologies, such as non-critical CHD and respiratory and infective illnesses, is an additional advantage ... ${ }^{[14]}$ 


\section{Objectives}

The primary objective of this study was to test and document the implementation feasibility of POx screening at Mowbray Maternity Hospital (MMH), a busy level 2 maternity hospital in the Western Cape Province, SA. The majority of infants in SA are born in similar or lower-level care settings. We also assessed nursing and parental acceptance. Our secondary aims were to determine the number and spectrum of CCHD and other significant illnesses identified and to report sensitivity and specificity of POx screening using Nellcor Oximetry technology (currently used in most Western Cape state facilities).

\section{Methods}

This was a prospective pilot study of implementation of predischarge newborn POx screening at sea level, following informed parental consent, in our busiest $\mathrm{MMH}$ postnatal ward. The study period was from 19 May to 19 September 2014. Ethics approval was granted from the Human Research Ethics Committee, Faculty of Health Sciences, University of Cape Town (HREC Ref. 661/2013) and from the Mowbray Maternity Hospital Research Committee.

\section{Setting}

MMH is a secondary-level maternity hospital serving several local midwife obstetric units (MOUs) as well as the surrounding area. Patients are referred from the Mitchell's Plain, Gugulethu, Retreat and Hanover Park MOUs and False Bay Hospital, therefore representing the bulk of medium-risk pregnancies in our region. Of the $\sim 38000$ births in the Metropole West region of the Western Cape each year, 11400 (about one-third) are facilitated at $\mathrm{MMH}$, which during 2014 had 950 deliveries a month on average.

One postnatal ward, B ward, was selected because it is MMH's busiest postnatal ward, which at the time of the study received $\sim 40 \%$ mothers after normal vaginal deliveries, who may be discharged after 6 hours, and $\sim 60 \%$ after caesarean section deliveries, discharged at 72 hours or later. B ward generally received the 'sicker' mothers or those with more complications, and discharged an average of 570 babies per month, 19 per day. During our study period, with general staff shortages, B ward had an average of 5 staff members per 12-hour shift ( 3 sisters, 1 staff nurse and 1 enrolled nursing assistant).

\section{Training and consent}

All Mowbray staff shifts received POx education and practical training. As per protocol

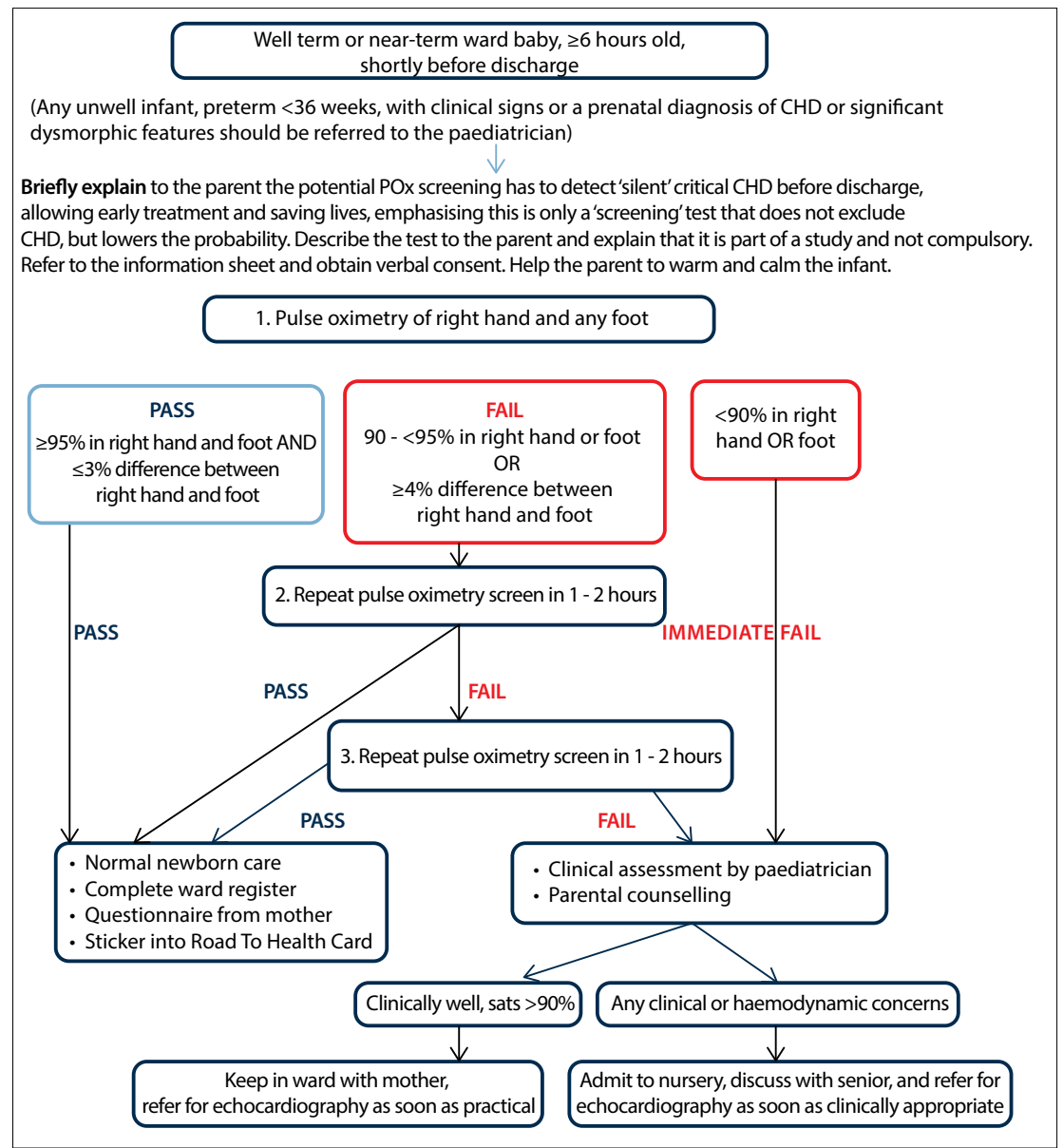

Fig. 1. POPSICLe study protocol. (Adapted from the proposed protocol in Kemper et al..[12] similar to Ewer et al.'s ${ }^{[14]}$ Health Technology Assessment protocol and with advice from Anne de-Wahl Granelli.)

(Fig. 1), all saturations $<90 \%$ were to be interpreted as an 'immediate fail' and the paediatric medical officer was to be called to review the baby. Saturations of $>90-<95 \%$, as well as arm-leg saturation differences of $\geq 4 \%$, required a second screen.

The mothers admitted to $\mathrm{B}$ ward received a POx pamphlet in their own language (English/Afrikaans/Xhosa). The staff member allocated to identify and process the discharges for that day did room-to-room parent education, explaining POx screening and the study. If a mother verbally consented, the screening was done at the bedside with ample opportunity for the mother to ask questions. A detailed study register was kept, detailing the baby's screening age, the saturations measured, how long it took, and comments. After screening, the mothers were asked to fill in a questionnaire. Nurses were asked to complete an anonymous questionnaire and to comment on the screening procedure.

\section{Inclusion and exclusion criteria}

All neonates $>6$ hours old with no clinical signs of cardiovascular disease were eligible for study enrolment. 'Unwell' infants, those $<6$ hours old, or those born to mothers $<14$ years of age or unable to give informed verbal consent (due to illness, illiteracy or language barriers) were not eligible. All infants with a prenatal diagnosis of CHD or any signs of CHD, including a heart murmur $(\geq 3 / 6)$ or significant dysmorphic features, were excluded.

All babies screened had a sticker placed on their Road To Health Card noting that POx screening had been performed, with a request to contact the investigators in the event of a subsequent diagnosis of CHD. Local death registers, the Perinatal Problem Identification Programme database and the Red Cross War Memorial Children's Hospital cardiology database were checked for any false negatives up to 9 months after the end of the study.

\section{Statistical analysis}

Data were captured in a detailed study register, and transferred to an Excel spreadsheet (2010, Microsoft, USA) and subsequently to Stata 11.2 (StataCorp, USA) for analysis. The $95 \%$ confidence intervals (CIs) were 
Table 1. POPSICLe need for second and third screens, or echocardiography

\begin{tabular}{|c|c|c|c|}
\hline & 2nd screen needed & 3rd screen needed & Echocardiograms \\
\hline Patients, $n$ & 18 & 3 & 2 \\
\hline \multirow[t]{2}{*}{ Reason/nurse's comment } & 15 passed on 2 nd screen & 2 technical issues (new probe needed) & 1 TGA (immediate fail) \\
\hline & 3 went on to 3 rd screen & $\begin{array}{l}1 \text { appropriate, failed } 3 \text { screens, assessed by paediatrician } \\
\text { and admitted to NICU }\end{array}$ & 1 sepsis/congenital pneumonia \\
\hline
\end{tabular}

calculated using the 'cii' command in Stata. Continuous variables were summarised as means (standard deviation) or medians (interquartile range (IQR)), and categorical data using frequency and percentages. We also computed the diagnostic odds ratio (OR), which is a single measure of effectiveness of a diagnostic test that is independent of prevalence. A diagnostic OR of $>1$ is indicative of a discriminatory test, whereas a diagnostic OR of $>400$ indicates an acceptable effect size. ${ }^{[15]}$

\section{Results}

Nursing staff were able to approach 1017 mothers of the 2256 babies discharged during the study period. We were therefore able to offer predischarge saturation screening to $45.1 \%$ of all B-ward infant discharges. Of these mothers, 1001 (98.4\%) consented to be included.

A total of 1001 babies were screened, at a median age of 60 hours (IQR $36-77$ ). Thirty (3.0\%) were $>5$ days old and 104 $(10.4 \%)$ were $<24$ hours old. The latest screen was at 170 hours of age and the earliest at 7 hours. There was no significant difference between saturation values of babies tested at $<24$ hours (mean 97.\%), $\geq 24-120$ hours $(97.8 \%)$ or $>120$ hours $(98.1 \%)$.

Of the 1001 babies screened, only 18 (1.8\%) needed a second screen. Four of the second screens should have been immediate fails' because one saturation was $<90 \%$ ( 86 $88 \%$ ), but all passed on the second screen. These were counted as 'protocol errors'. Three third screens $(0.3 \%)$ were needed, 2 owing to technical issues (probe needed replacement), while 1 baby was appropriately admitted to the neonatal intensive care unit (NICU) with pneumonia/septicaemia (Table 1).

The ward register comments showed that $97.8 \%$ of the tests were perceived as 'easy' to perform. These babies were described as calm, asleep or breastfeeding. Five babies were noted to be crying, but the test was still easy to perform. Twenty-four tests $(2.3 \%)$ were difficult to perform: 16 babies were restless or crying, there was a technical issue in 7 cases (difficulty with probe placement, probe or machine malfunction), and 1 case was assessed as difficult because the

Table 2. POPSICLe screening time assessment

\begin{tabular}{|c|c|c|c|c|}
\hline & \multicolumn{3}{|c|}{ Time screen took (min) } & \multirow[b]{2}{*}{ Total } \\
\hline & $<3$ & $3-5$ & $>5$ & \\
\hline Screens, $n$ & 961 & 44 & 17 & 1022 \\
\hline \multirow[t]{5}{*}{$\begin{array}{l}\text { Reason/nurse's } \\
\text { comment }\end{array}$} & $\begin{array}{l}\text { Calm/feeding/ } \\
\text { sleeping/easy }\end{array}$ & 7 crying/restless & 3 crying a lot & \\
\hline & & 4 technical issues & 5 technical issues & \\
\hline & & 33 easy/calm & 6 easy/calm & \\
\hline & & $\begin{array}{l}1 \text { immediate fail, } \\
\text { admitted }\end{array}$ & $\begin{array}{l}3 \text { calm but difficult } \\
\text { (1 failed } 3 \text { screens, admitted) }\end{array}$ & \\
\hline & & $\begin{array}{l}11 \text { failed first/second } \\
\text { screens }\end{array}$ & $\begin{array}{l}10 \text { failed first/second } \\
\text { screens }\end{array}$ & \\
\hline
\end{tabular}

baby failed all three screens and needed admission.

In total, 1022 screens were done: 961 $(94.0 \%)$ took $<3$ minutes to perform, while $44(4.3 \%)$ took between 3 and 5 minutes. Of these, 11 were failed first or second screening tests. Seventeen tests (1.7\%) took $>5$ minutes to perform: 3 took long because the baby was crying or restless, in 5 there were technical problems with probe or machine malfunction, and 1 infant failed all three tests and was admitted (Table 2).

Thirty-one protocol errors (3.1\%) were made: 4 were a second screen that should have been an immediate fail, and all the infants passed on the second screen. Seventeen errors were recorded because saturation measurements of 90 - $94 \%$ were accepted as a pass, and 10 because a difference of $\geq 4 \%$ was accepted as a pass where arm-leg saturations were 96 - 100\% $(n=4), 95$ - 99\% ( $n=1), 95$ $100 \%(n=1), 100-95 \%(n=3)$ and $99-95 \%$ $(n=1)$. All of the error cases were followed up and all the babies were well, except for one twin who died aged 3 months in the Congo with 'coughing.'

Only two patients $(0.2 \%)$ failed the screening, were admitted to the NICU and had an echocardiogram. The first was an immediate fail on the first screen, and the echocardiogram revealed a transposition of the great arteries (TGA). Oral prostaglandin was started and she was transferred to a cardiac centre in good condition, where she had a successful arterial switch operation the following day with no complications. The other patient failed three screens and was admitted to the NICU with respiratory distress and elevated septic markers. He required 3 days of nasal continuous positive airway pressure ventilation (nCPAP) and 7 days of intravenous antibiotics. The echocardiogram was normal. Feedback from the local tertiary paediatric cardiology service revealed that one patient with a coarctation of the aorta was missed (false-negative rate $0.09 \%$ ) by our screening; the patient presented to the Red Cross War Memorial Children's Hospital emergency room in an unstable condition at the age of 6.5 weeks (Fig. 2).

In this pilot study, the sensitivity was $50 \%(95 \%$ CI 1.3 - 98.7) and the specificity 99.9\% (95\% CI 99.4 - 100), with a test utility (\% correct) of $99.8 \%$. The calculated diagnostic OR was 999.

During the study period, another three babies were clinically diagnosed with significant cardiac lesions (tetralogy of Fallot, atrioventricular septal defect (AVSD), double-outlet right ventricle with ventricular septal defect and pulmonary stenosis) from the same ward, which excluded them from screening. The incidence of CCHD in $\mathrm{B}$ ward over the study period was therefore 4 per 1000 , the AVSD being a significant cardiac lesion but not critical. The one error case in which a twin died of 'coughing' in the Congo at the age of 3 months may also have been severe CHD. 


\section{Feedback from nurses}

All the nurses involved were satisfied that the purpose and aims of the study were easy to understand and the study protocol was easy to follow. All but one nurse felt confident and able to explain the purpose and limitations of screening adequately after the training sessions. Three nurses said that they would have preferred a paediatrician to explain the process to the parents. Only one nurse found the screening difficult to perform. Most (91.6\%) of the nursing feedback indicated that the nurses did not have enough time to perform the screening as well as complete their routine required tasks. One nurse commented specifically that the study screening time was increased owing to the paperwork required for the study, which would be less if screening was routine. Half of the nurses felt that it would be possible to introduce screening into everyday routine at MMH. However, 25\% felt it would only be possible if adequate nursing staff were available. Another 25\% felt it would not be possible with the current staffing allocation.

\section{Feedback from parents}

We received feedback from 726 patients, 16 who refused screening and 710 who consented. Of those who refused, 4 stated they were too anxious about possible outcomes of the screening. One mother refused because she felt overwhelmed by the numerous routine procedures that she and her baby had been subjected to. One mother was uncomfortable with the idea of being involved in a study, and another felt confident that her child was healthy and did not feel that further tests were necessary. The remaining mothers did not give reasons for their refusal to participate. Two nurses observed that mothers who refused testing did so because they were eager to leave the hospital and were worried that screening might have meant staying longer.

Of the 710 screened patients who filled in the parent's questionnaire, 700 (98.6\%) stated that the test was adequately explained, the time taken acceptable, and the screening as a whole acceptable and beneficial to their child's and other children's health. Interestingly, two of these patients felt that the screening was too quick and that more time should have been taken. Forty mothers commented specifically that screening was worthwhile and that they appreciated their babies being screened. Four mothers commented that screening should be offered to all babies. Eight mothers (1.1\%) felt the time taken was too long. Two mothers $(0.3 \%)$ said that, although they had consented,

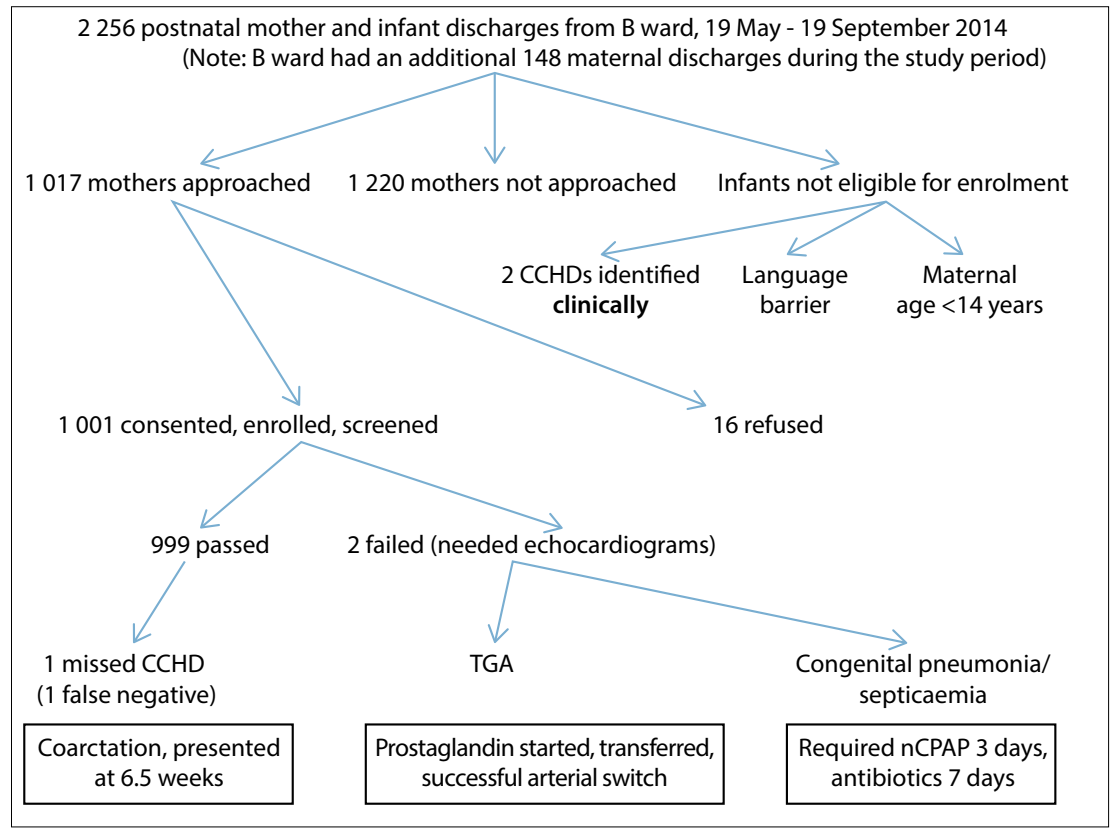

Fig. 2. POPSICLe study findings.

screening was of no benefit to their child. In summary, $98.6 \%$ of mothers who consented to their babies receiving screening felt that the test was acceptable and beneficial.

\section{Discussion}

Our study documents the practical feasibility of newborn POx screening for CCHD in a large public sector maternity hospital in SA, plus provider and parental attitudes to it. We demonstrated acceptance from parents and staff, and an appropriate sensitivity and a high specificity for POx screening at sea level. Our pilot study of 1001 patients showed a sensitivity of $50 \%$ and specificity of $99.9 \%$, and one false-positive case with neonatal sepsis. This compares well with Thangaratinam et al. ${ }^{[11]}$ systematic review and meta-analysis of 229421 babies screened, which showed an overall sensitivity of $76.5 \%$, specificity of $99.9 \%$ and false-positive ratio of $0.14 \%$. Our lower sensitivity reflects our small study number and only two patients failing the screen, one with a true CCHD and one with neonatal sepsis. This low incidence probably also reflects our good clinical pickup of CHD, these cases being excluded from the study. Our hospital is viewed as one of the better-resourced secondary-level hospitals in the country, where a doctor reviews all infants daily. However, in terms of nursing staff, severe staffing resource challenges during our study period resulted in our only offering screening to $45.1 \%$ of babies discharged from the study ward. Yet the diagnostic ability of the test was significant, with high specificity, diagnostic odds and percentage correct.
We postulate that routine screening, not part of a study, would translate into less nursing time spent explaining, consenting and completing paperwork, which may result in a higher percentage screened. We did not employ any additional staff, so the nurse doing the screening was required to perform all the necessary explanations, consent and paperwork required by the study. During our study period, we discovered that an average of five nursing staff per shift had to discharge an average of 19 babies and 20 mothers per day, in addition to their routine care of inpatients in a 46-bed ward with a bed occupancy rate of $98-100 \%$. This represents an enormous amount of work. We suggest that with a full complement of nurses or one extra nurse per shift, routine POx screening could be implemented at MMH with much better coverage.

Despite severe staff shortages, the everincreasing patient-to-staff ratio and increasing client expectations, our nursing staff viewed POx screening favourably and were eager to see it implemented routinely. Our study also confirmed parental acceptance. Most of the mothers from whom feedback was obtained felt that routine screening should be standard practice.

Many studies have documented the benefits and feasibility of POx screening in developed countries. ${ }^{[8-10,13]}$ SA and lesserresourced countries, however, face many barriers to implementation of routine POx screening. Most of these barriers stem from an underfunded health service caring for an ever-increasing patient load. This translates to fewer staff (medical and nursing) hours 
and less equipment available than is desirable for the clinical service required.

Almost all birthing facilities in the Western Cape are in the process of obtaining saturation monitors, since these are now deemed essential in neonatal resuscitation and neonatal care. POx training for all staff will therefore be essential and POx monitors will become available everywhere. POx did prove simple enough to implement in our setting. With regard to cost, probes are relatively cheap and in our study practically proved to be re-usable multiple times.

Our study's two echocardiograms per 1001 babies debunk the argument that POx screening will add to the burden of an already overloaded cardiac service. Our local cardiac service welcomes early diagnosis of CHD, which decreases the mortality, morbidity and cost associated with late referrals.

\section{Study limitations}

Firstly, the study was done only at one institution. Staffing and equipment availability may be very different at other birthing facilities. However, being one of the busiest birthing facilities and the site of nearly one-third of the births in the Western Metropole, MMH may still be a good representation of our area in the Western Cape, and with our nursing staff shortages experienced, we probably compare well with most Western Cape hospitals. Secondly, our study was a pilot done in only one postnatal ward, our busiest. Other 'quieter' postnatal wards may have had a higher screening rate. Thirdly, since this was an implementation feasibility study with no control group and no physical follow-up, interpretation of our specific case findings is of limited value. Potential false negatives may not have been fed back to the investigators, undermining the accuracy of this screening, although all efforts were made to trace any 'missed' cases and POx screening accuracy has already been proven internationally. Lastly, interpretation of staff or parents' comments may be subjective, but the written anonymous questionnaires showed acceptance and an overwhelmingly favourable response.

\section{Implications for practice}

'Implementation feasibility' means a high success rate of screening along with sufficiently low barriers and resource drain. The screening success rate attained has been discussed. In our study the anticipated barriers of increased cardiology service workload, cardiac care costs, high equipment expense and lack of acceptance by parents and nursing staff were not encountered. Our study also showed that the age at which screening is done (as long as it is $>6$ hours after birth) is not relevant when tested at sea level. This is in keeping with the findings of most large multicentre studies and reconfirms the practical potential for all newborns to have predischarge screening even when discharged at 6 hours after birth. The only significant barrier was a shortage of nursing staff time, which unfortunately is a reality in the majority of health facilities in SA. We postulate that routine predischarge screening, not part of a study and translating to less nursing time per screen, will lead to a higher screen rate. Also, the ongoing controversy over whether both pre- and postductal screening is significantly superior to a single postductal screen ${ }^{[16]}$ may mean that a single foot saturation screen before discharge is the more practical solution in resource-limited settings.

\section{Conclusion}

POx screening was accepted and supported by both staff and parents. We believe that if not part of a study, but done as a routine and with adequate nursing staff, it could be implemented successfully with a high screen rate and without excessive false positives or errors. Our study confirms that POx screening does not significantly increase the paediatric cardiology service workload. Earlier diagnosis and management should reduce morbidity and ultimately reduce cost and workload.

Acknowledgements. We are grateful to the mothers and infants who participated in the study. Sincere appreciation goes to the nursing staff of MMH. This work was funded in part by the School of Child and Adolescent Health Research Committee, Department of Paediatrics, Red Cross War Memorial Children's Hospital and University of Cape Town.

\section{References}

1. Hoffman JI, Kaplan S. The incidence of congenital heart disease. J Am Coll Cardiol 2002;39(12):18901900. DOI:10.1016/S0735-1097(02)01886-7

2. Botto LD, Correa A, Erickson JD. Racial and temporal variations in the prevalence of heart defects. Pediatrics 2001;107(3):e32-e32. DOI:10.1542/peds.107.3.e32

3. Zheleva B. Linked by a common purpose: Global efforts for improving pediatric heart health: A report by Children's Heart Link. Cong Cardiol Today 2007;5(7):1-8.

Hoffman JI. The global burden of congenital heart disease: Review article. Cardiovasc J Afr 2013;24(4):141-145. DOI:10.5830/CVJA-2013-028

5. Mahle WT, Newburger JW, Matherne GP, et al. Role of pulse oximetry in examining newborns for congenital heart disease: A scientific statement from the AHA and AAP. Pediatrics 2009;124(2):823836. DOI:10.1542/peds.2009-1397

6. Hewitson J, Zilla P. Children's heart disease in sub-Saharan Africa: Challenging the burden of disease: Children's heart disease. SA Heart 2010;7(1):18-29. http://www.pcssa.org/test/wp-content/ disease: Children's heart disease. SA Heart 2010;7(1):18-2
uploads/2013/12/JH-P-Zilla.pdf (accessed 1 July 2016).

Valmari P. Should pulse oximetry be used to screen for congenital heart disease? Arch Dis Child Fetal Neonatal Ed 2007;92(3):F219-F224. DOI:10.1136/adc.2005.090282

Neonatal Ed 2007;92(3):F219-F224. DOI:10.1136/adc.2005.090282 detection of duct dependent congenital heart disease: A Swedish prospective screening study in 39,82 newborns. BMJ 2009;338:a3037. DOI:10.1136/bmj.a3037

9. Ewer AK, Middleton LJ, Furmston AT, et al. Pulse oximetry screening for congenital heart defects in newborn infants (PulseOx): A test accuracy study. Lancet 2011;378(9793):785-794. DOI:10.1016 S0140-6736(11)60753-8

10. Riede FT, Wörner C, Dähnert A, Möckel A, Kostelka M, Schneider P. Effectiveness of neonatal pulse oximetry screening for detection of critical congenital heart disease in daily clinical routine - results from a prospective multicenter study. Eur J Pediatr 2010;169(8):975-981. DOI:10.1007/s00431-010$1160-4$

11. Thangaratinam S, Brown K, Zamora J, Khan KS, Ewer AK. Pulse oximetry screening for critical congenital heart defects in asymptomatic newborn babies: A systematic review and meta-analysis. congenital heart defects in asymptomatic newborn babies: A systematic

12. Kemper AR, Mahle WT, Martin GR, et al. Strategies for implementing screening for critical congenital Kemper AR, Mahle WT, Martin GR, et al. Strategies for implementing screening for
heart disease. Pediatrics 2011:128(5):e1259-e1267. DOI:10.1542/peds.2011-1317

13. Meart disease. Pediatrics 2011;128(5):e1259-e1267. DOI:10.1542/peds.2011-1317
A, Brügman-Pieper S, Due R, et al. First day of life pulse oximetry screening to detect Meberg A, Brügman-Pieper S, Due R, et al. First day of life pulse oximetry screenin
congenital heart defects. J Pediatr 2008;152(6):761-765. DOI:10.1016/j.jpeds.2007.12.043

14. Ewer AK, Furmston AT, Middleton LJ, et al. Pulse oximetry as a screening test for congenital heart defects in newborn infants: A test accuracy study with evaluation of acceptability and costeffectiveness. Health Technol Assess 2012;16(2). DOI:10.3310/hta16020

15. Zhou XH, Obuchowski NA, McClish DK. Statistical Methods in Diagnostic Medicine (Wiley Series in Probability and Statistics). 2nd ed. New York: Wiley, 2011.

16. Narayen IC, Blom NA, Ewer AK, Vento M, Manzoni P, te Pas AB. Aspects of pulse oximetry screening for critical congenital heart defects: When, how and why? Arch Dis Child Fetal Neonatal Ed 2016;101(2):F162-F167. DOI:10.1136/archdischild-2015-309205

Accepted 8 June 2016. 